\title{
Highlights of Pharmacist Roles in Hematopoietic Cell Transplantation and Cellular Therapy
}

\author{
Ho Ying Shi', Trai Tharnpanich², Bo Yu
}

'Department of Pharmacy, Singapore General Hospital, Singapore, ${ }^{2}$ Faculty of Pharmaceutical Sciences Chulalongkorn University, Bangkok, Thailand, '3Department of Pharmacy, and Clinical Research Institute Office, Tongren Hospital, Shanghai Jiao Tong University, School of Medicine, Shanghai, China

\begin{abstract}
Patients with hematological malignancies receiving hematopoietic stem cell transplantation (HSCT) or chimeric antigen receptor (CAR) T-cell therapy are known to have complex pharmacotherapy. To ensure safe and effective care in preventing and managing drug-related problems, pharmacists trained in HSCT and CAR T-cell therapy fulfill various roles in providing care for these patients. With polypharmacy and complex medication regimens, drug interactions are one of the major aspects that pharmacists review regularly to ensure the efficacy of treatment while minimizing toxicity. Pharmacists anticipate various complex drug interactions, understanding the possible mechanisms of drug interactions, and manage these complex drug regimens in patients undergoing HSCT and CAR T-cell therapy. In addition, antimicrobial prophylaxis is also important supportive care for immunocompromised patients. Pharmacists are capable of evaluating the risk of opportunistic infections, anticipating the type of antimicrobial prophylaxis required, and setting a policy with a robust antimicrobial regime. This can promote consistency in patient care and improve patient outcomes in terms of morbidity and mortality associated with opportunistic infections. Lastly, pharmacists are equipped with a skillset to ensure a seamless transition of care, provide education for patients and healthcare providers, promote medication adherence, and contribute to research and quality improvement.
\end{abstract}

Key words pharmacist, drug interactions, antimicrobial prophylaxis, transition of care, hematopoietic stem cell transplantation

Submitted August 20, 2021; Accepted August 20, 2021; Published online October 14, 2021; Issued online October 14, 2021

Correspondence: Ho Ying Shi, Singapore General Hospital, 1 Hospital Drive Singapore 169608 Block 7 Level 2 Hematology Pharmacy, Email: ho.ying.shi@sgh.com.sg

This article was created from selected presentations at the 26th Annual Congress of APBMT and was handled by Guest Editor Jeff Szer before submission.

\section{Introduction}

Hematopoietic stem cell transplantation (HSCT) and CAR T-cell therapy are actively performed for the treatment of many hematological malignancies ${ }^{1}$. Patients with hematological malignancies receiving HSCT and CAR T-cell therapy are known to have very complex pharmacotherapy. Multidisciplinary collaboration among pharmacists, physicians, nurses, and coordinators is of paramount importance to ensure safe and effective care in preventing and managing drug-related problems. Pharmacists who are trained in HSCT and CAR T-cell therapy fulfill various roles in providing care for the pa- tient population, including direct patient care, medication management, policy development, transition of care, patient and staff education, supportive care, and quality tracking ${ }^{2}$.

In this section, we highlight three important aspects that pharmacists are actively involved in for the care of patients undergoing HSCT and CAR T-cell therapy. First, we reviewed clinical data describing clinically important pharmacokinetic drug interactions and provided recommendations for preventing and/or managing these drug interactions. Next, we focused on the indications and options for antimicrobial prophylaxis in patients receiving chimeric antigen receptor (CAR) T-cell therapy. 
Lastly, we explored the various contributions of pharmacists as drug experts in specific areas of transition of care, education, medication adherence, and research.

\section{Management of Common Drug Interactions in HSCT Patients}

Patients undergoing HSCT are often treated with polypharmacy, a complex medical regimen consisting of at least five drugs. Various combinations of chemotherapy, immunosuppressants, and antimicrobial drugs have the potential for multiple adverse drug interactions due to the narrow therapeutic windows and high toxicity profiles of these drugs ${ }^{3}$.

Drug interactions can occur through pharmacokinetic or pharmacodynamic interactions. Pharmacokinetic interactions may occur at the level of absorption, distribution, metabolism, and elimination. Drug interactions are difficult to interpret, given the great disparities in how these interactions are defined and the wide spectrum of their severity among patients. Although some drug interactions may be anticipated based on a theoretical basis, not all interactions are clinically significant ${ }^{4,5}$. Poor nutritional status, renal and liver dysfunction, and altered protein binding are known to potentiate the risk of clinically significant drug interactions. Morbidity increases when drug interactions lead to unexpected outcomes, including treatment failure ${ }^{6,7}$.

In this section, we review the potential mechanism and management of clinically important drug interactions that transplant physicians and pharmacists should know in order to reduce toxicity and maintain the efficacy of the intended treatment.

\section{Phenytoin and calcineurin inhibitors}

Phenytoin is an anti-epileptic drug prescribed for busulfan-based conditioning regimens in HSCT to prevent busulfan-induced seizures. Ciclosporin and tacrolimus are calcineurin inhibitors used in HSCT to prevent graft-versus-host disease (GVHD). As a CYP3A4 inducer, phenytoin is expected to have a significant interaction with CYP3A4 substrates, such as calcineurin inhibitors. Maintaining the trough level of calcineurin inhibitors within narrow therapeutic windows is not an easy task and requires pre-emptive management and close monitoring. Sub-therapeutic levels of cyclosporine at the earlier phase of HSCT are known to increase the risk of GVHD and early graft failure ${ }^{8}$.

Strategies to avoid this significant drug interaction have been adopted, including the use of non-interacting anti-epileptic medicines (AEMs) such as levetiracetam, given its favorable drug interaction profile ${ }^{8}$. While there are some concerns that this strategy may affect transplant outcomes, the study by Mccune et al. found no difference in the outcome of non-relapse mortality (NRM) or overall survival (OS) $)^{9,10}$. Despite the use of alternative AEMs to prevent busulfan-induced seizures in HSCT recipients, the acceptance has been slow, as most studies using busulfan-based conditioning were conducted with phenytoin ${ }^{11}$. Therefore, the strategy of adopting alternative AEMs is more feasible in institutions that have the facility to support busulfan therapeutic drug monitoring (TDM), to ensure that patients receive the intended busulfan plasma area under the curve $^{12}$.

Another strategy proposed to manage this significant drug interaction is to dose cyclosporine at a higher starting dose to ensure that ciclosporin achieves therapeutic levels by the day of stem cell infusion. In the study by Ho et al., patients receiving phenytoin as prophylaxis for busulfan-induced seizures were initiated at a higher starting dose of intravenous cyclosporine (4 $\mathrm{mg} / \mathrm{kg} /$ dose twice daily instead of $3 \mathrm{mg} / \mathrm{kg} / \mathrm{dose}$ twice daily). This higher starting dose resulted in a higher proportion of patients with therapeutic cyclosporin, without an increase in cyclosporin-related adverse events ${ }^{13}$.

\section{Azoles and calcineurin inhibitors}

Azoles are a group of antifungals commonly used to prevent or treat fungal infections in patients undergoing $\mathrm{HSCT}^{14}$. Azoles are metabolized by CYP3A4 isoenzymes and are strong inhibitors of CYP3A4 or CYP2C9, resulting in many potential drug interactions. One of the most significant drug-drug interactions in the HSCT setting is between azoles and calcineurin inhibitors, which can increase the serum levels of calcineurin inhibitors ${ }^{7}$. In view of the narrow therapeutic window of calcineurin inhibitors, TDM of calcineurin should be routinely conducted to adjust the dose of calcineurin inhibitors. More importantly, the degree of drug interactions may be affected by the route of administration of calcineurin inhibitors. For instance, ciclosporin has a large inter- and intra-individual variability in bioavailability due to differences in absorption and first-pass effects during oral administration. In the absence of azoles, the conversion ratios adopted for ciclosporin or tacrolimus from intravenous to oral formulations were 3:1 and 2:1, respectively. However, when used in combination with azoles, the degree of CYP3A 4 inhibition may differ; hence, the conversion ratios are changed to a lower ratio depending on the azole used to prevent supra-therapeutic concentration of calcineurin inhibitors, which may increase the risk for toxicity, such as nephrotoxicity and hepatotoxicity ${ }^{15,16}$. 


\section{Managing Patients Receiving CAR-T Cell Ther- apy from a Pharmacists Perspective: Our Experi- ence at Singapore General Hospital}

Patients receiving chimeric antigen receptor (CAR) Tcell therapy are at an increased risk of opportunistic infections due to the cumulative effect of prior treatments. In addition to cytokine release syndrome (CRS) and IEC-associated neurotoxicity syndrome (ICANS), infections are one of the most common side effects associated with CAR T-cell therapy, due to prolonged and protracted neutropenia in this patient population ${ }^{17}$. However, there are no clear recommendations on antimicrobial prophylaxis in CAR-T patients, and most studies adopted standard practices extrapolated from the allogeneic hematopoietic stem cell transplantation (alloHSCT) population.

In this section, we reviewed the antimicrobial prophylaxis that should be considered in patients undergoing CAR T-cell therapy. The current practices for antimicrobial prophylaxis at Singapore General Hospital (SGH) were derived from the European Society for Blood and Marrow Transplant (EBMT) and the Joint Accreditation Committee of ISCT and EBMT (JACIE), international guidelines, and best practices around the world $^{17-20}$.

\section{Anti-bacterial prophylaxis}

Given the anticipated prolonged neutropenia in patients receiving CAR T-cell therapy, we advocate for antibacterial prophylaxis with fluoroquinolone until patients are out of neutropenia. The drug of choice for $\mathrm{SGH}$ is oral ciprofloxacin, administered at $500 \mathrm{mg}$ twice daily ${ }^{17-20}$.

\section{Anti-viral Prophylaxis}

In line with the EBMT recommendation, all patients receiving CAR T-cell therapy at SGH receive prophylaxis for herpes virus, starting from lymphodepleting (LD) conditioning until one-year after CAR T-cell infusion and/or until the CD4+ count $>0.2 \times 10^{9} / \mathrm{L}(>200$ cells $/ \mathrm{mm}^{3}$ ). The drug of choice for SGH is oral acyclovir at a dose of $800 \mathrm{mg}$ twice daily ${ }^{17-20}$. After 30 days, frequently observed viral infections included respiratory viral infections, cytomegalovirus viremia (CMV), and pneumonia ${ }^{17}$. However, there is no recommendation concerning the frequency of CMV monitoring after CAR T-cell therapy. Hence, we propose CMV PCR monitoring every 1-2 weeks, starting 30 days after CAR-T cell infusion.

\section{Anti-pneumocystis prophylaxis}

All patients receiving CAR T-cell therapy should receive prophylaxis against Pneumocystis carinii jiroveci
(PCP), as lymphodepleting conditioning typically consists of purine analogs such as fludarabine. Prophylaxis should commence following recovery from neutropenia or around day +21 if neutropenia persisted, at the discretion of the attending physician. Our drug of choice at $\mathrm{SGH}$ is oral co-trimoxazole, to be administered at least until one-year after CAR T-cell infusion and/or until the CD4+ count $>0.2 \times 10^{9} / \mathrm{L}\left(>200 / \mathrm{mm}^{3}\right)$. For patients with contraindications to co-trimoxazole, the alternative would be intravenous pentamidine or oral dapsone ${ }^{17-20}$

\section{Anti-fungal prophylaxis}

The use of antifungal prophylaxis in patients receiving CAR-T cell therapy is less straightforward and dependent on the patients' risk factors. At SGH, the preferred primary antifungal prophylaxis is oral fluconazole. At-risk patients (defined as prior allogeneic HSCT, prior invasive aspergillosis, or receiving corticosteroids) should receive oral posaconazole. However, in patients who are already on treatment for pre-existing fungal infections, the choice of antifungal agents for secondary prophylaxis should be made in consultation with an infectious disease physician ${ }^{17-20}$. Additionally, considering the potential drug-drug interactions between cyclophosphamide active metabolites and azoles, we recommend the initiation of antifungal prophylaxis after the completion of lymphodepleting conditioning or on day zero of CAR T-cell therapy, and to continue until the recovery from neutropenia ${ }^{21}$.

\section{Use of granulocyte colony stimulating factors (G- CSF)}

The use of G-CSF in patients receiving CAR-T cell therapy is controversial. The survey conducted by ASBMT showed that there were varying practices among 28 respondents on the use of G-CSF in patients receiving CAR T-cell therapy. About $46 \%$ used G-CSF according to product labeling, 29\% never administered growth factors, $14 \%$ determined the use of growth factors on a patient-specific basis, and $11 \%$ administered growth factors to all patients ${ }^{20}$. Conventionally, G-CSF has been postulated to increase the risk of CRS and ICANS $^{22}$. However, Galli et al. reported that prophylactic administration of G-CSF in patients with grade 4 neutropenia did not increase the risk of severe CRS or ICANS. GCSF administration did not interfere with CAR T-cell expansion kinetics while preserving CAR-T anti-lymphoma activity with no impact on the quality of responses and outcomes ${ }^{23}$. Considering the varied practices in a relatively new therapy, the consensus at SGH is to consider the use of G-CSF after day 5 of CAR Tcell infusion, only if clinically indicated, and there is no ongoing CRS/ICANS. 


\section{Hypogammaglobulinemia}

Monitoring of serum immunoglobulin is crucial because of B cell aplasia and hypogammaglobulinemia caused by CAR T-cell therapy. Hence, we propose intravenous immunoglobulin (IVIg) replacement at a dose of $0.25 \mathrm{~g} / \mathrm{kg}$ when serum $\operatorname{lgG}$ level is $<400 \mathrm{mg} / \mathrm{dL}$ or $<4 \mathrm{~g} / \mathrm{L}^{24}$.

\section{Pharmacist Role in HSCT Unit: China Experi- ence}

HSCT pharmacists play an important role in the HSCT unit at Shanghai Tongren Hospital, as they oversee and manage a wide range of pharmacy services to cater for patients with complex clinical trajectories. In this session, we will focus on the role of pharmacy services for patients who undergo HSCT in various aspects, including the transition of care, education, medication adherence, and research.

\section{Transition of care}

The transition of care involves the coordination and continuity of healthcare. Critical points of transitions include admission and discharge from hospital, escalation, and de-escalation of care to and from intensive care units, and transitions to home or step-down care facilities. The transition of the care environment is a major risk factor for medication errors due to the lack of communication between healthcare providers, missed follow-ups of patients, inadequate medication reconciliation, and the absence of patient involvement in medication management. Inconsistent care coordination contributes to a readmission rate of $20 \%$ within 30 days of discharge ${ }^{25}$. At Shanghai Tongren Hospital, HSCT pharmacists can provide support in the risk management of patients in terms of medication reconciliation, medication adherence, and patient education.

\section{Education}

Education for HSCT and cellular therapy should be carried out not only for patients but also for caregivers. The contents should include but are not limited to, instructions to take the medications correctly Pharmacists also educate patients about potential drug-drug/drugfood/drug-herb interactions. In addition to helping shape new programs for HSCT-related pharmacy services, HSCT pharmacists also hold several key academic positions and provide pharmacy training all around China. Furthermore, education also extends to the training of healthcare providers and trainees, in various pharmacist-led educational services, to optimize collaborative, patient-centered care focused on patient safety $^{26}$. At Shanghai Tongren Hospital, some examples of pharmacist-led training include safe handling of che- motherapy and supportive care management.

\section{Medication adherence}

The World Health Organization (WHO) describes medication adherence as the most important factor defining treatment efficacy ${ }^{27}$. Medication adherence is particularly challenging to reinforce in the setting of HSCT as post-transplant medications to prevent transplantassociated complications involving many drugs such as immunosuppressants, anti-infective drugs, and supplements (e.g., magnesium, folic acid). Adherence to medications has been reported to fall below the recommended $80 \%-95 \%$ in pediatric and adult HSCT recipients $^{28}$. Some patients are discouraged by the side effects of medications (e.g., magnesium-induced diarrhea). Others may not understand the rationale for antiinfective drugs when patients are often asymptomatic. However, it is known that non-adherence could potentially cause major and serious post-HSCT complications, such as GVHD and infections ${ }^{29}$. At Shanghai Tongren Hospital, pharmacists emphasize the importance of optimal medication adherence through constant engagement with patients and caregivers at various transitions of care.

\section{Research}

Pharmacists are also involved in the management of clinical trials involving patients receiving HSCT or cellular therapy. There is an increasing number of clinical trials related to HSCT and cellular therapy, such as the combination of novel treatments with HSCT and B-cell maturation antigen (BCMA)-targeted CAR-T cell therapy. The patients included in these clinical trials may have received multiple lines of therapy. Therefore, safety monitoring is extremely important, and pharmacists can help reduce or identify any potential harm or adverse effects associated with trial medications ${ }^{30}$.

\section{Conclusion}

Patients with hematological malignancies receiving HSCT or CAR T-cell therapy, are known to have very complex pharmacotherapy. Pharmacists are integral members of multidisciplinary teams aiming to ensure safe and effective care in the prevention and management of drug-related problems. In this review, we highlighted the key roles of pharmacists: 1) preventing and/ or managing drug interactions, 2) developing policies on antimicrobial prophylaxis to prevent opportunistic infections, and 3) providing education to improve medication adherence at various transitions of care. Beyond these core roles, pharmacists in the Asia-Pacific region are encouraged to build upon existing knowledge to improve pharmacy practice and patient outcomes. This can 
be done through pharmacist-led clinical services in areas such as long-term follow-up and palliative care, which has much to offer to patients undergoing HSCT and CAR T-cell therapy.

\section{Author Contributions}

TT, HYS, and BY contributed to the draft of manuscript. HYS wrote the final version of manuscript.

\section{Conflicts of Interest}

The authors declare no conflict of interest. Disclosure forms provided by the authors are available on the website.

\section{References}

1. Fuhr U. Improvement in the handling of drug-drug interactions. Eur J Clin Pharmacol. 2008; 64: 167-71.

2. Langebrake C, Admiraal R, van Maarseveen E, Bonnin A, Bauters T; EBMT Working Group. Consensus recommendations for the role and competencies of EBMT clinical pharmacist and clinical pharmacologist involved in hematopoietic stemc cell transplantation. Bone Marrow Transplantation. 2020; 55: 62-69.

3. Glotzbecker B, Duncan C, Alyea E 3rd, Campbell B, Soiffer R. Important drug interactions in hematopoietic stem cell transplantation: What every physicain should know. Biol Blood and Marrow Transplantation. 2012; 18: 989-1006.

4. Scripture CD, Figg WD. Drug interactions in cancer therapy. Nat Rev Cancer. 2006; 6: 546-58.

5. Riechelmann RP, Tannock IF, Wang L, Saad ED, Taback NA, Krzyzanowska MK. Potential drug interactions and duplicate prescriptions among cancer patients. J Natl Cancer Inst. 2007; 99: $592-600$.

6. van Leeuwen RW, Swart EL, Boven E, Boom FA, Schuitenmaker MG, Hugtenburg JG. Potential drug interactions in cancer therapy: a prevalence study using an advanced screening method. Ann Oncol. 2011; 22: 2334-41.

7. Mihara A, Mori T, Aisa Y, Yamazaki R, Iketani O, Tanigawara $\mathrm{Y}$, et al. Greater impact of oral fluconazole on drug interaction with intravenous calcineurin inhibitors as compared with intravenous fluconazole. Eur J Clin Pharmacol. 2008; 64: 89-91.

8. Rogosheske JR, Fargen AD, DeFor TE, Warlick E, Arora M, Blazar BR, et al. Higher therapeutic CsA levels early post transplantation reduce risk of acute GVHD and improves survival. Bone Marrow Transplant. 2014; 49: 122-5.

9. Eberly AL, Anderson GD, Bubalo JS, McCune JS. Optimal prevention of seizures induced by high-dose busulfan. Pharmacotherapy. 2008; 28: 1502-10.

10. Floeter AE, McCune JS. Levetiracetam for the prevention of busulfan-induced seizures in pediatric hematopoietic cell transplantation recipients. J Oncol Pharm Pract. 2017; 23: 344-9.

11. McCune JS, Wang T, Bo-Subait K, Aljurf M, Beitinjaneh A, Bubalo J, et al. Association of Antiepileptic Medications with Outcomes after Allogeneic Hematopoietic Cell Transplanta- tion with Busulfan/Cyclophosphamide Conditioning. Biol Blood Marrow Transplant. 2019; 25: 1424-31.

12. Palmer J, McCune JS, Perales MA, Marks D, Bubalo J, Mohty $\mathrm{M}$, et al. Personalizing Busulfan-Based Conditioning: Considerations from the American Society for Blood and Marrow Transplantation Practice Guidelines Committee. Biol Blood Marrow Transplant. 2016; 22: 1915-25.

13. Ho YS, Chong MF, Ng VC, Ho A, Ng HY. Higher Starting Dose of Ciclosporin Optimized Therapeutic Levels in Allogeneic Hematopoietic Stem Cell Transplant Patients Receiving Phenytoin for Busulfan-induced Seizure Prophylaxis. Blood Cell Therapy. 2021; 4: 58-64.

14. Baden LR, Swaminathan S, Angarone M, Blouin G, Camins $\mathrm{BC}$, Casper C, et al. Prevention and Treatment of CancerRelated Infections, Version 2.2016, NCCN Clinical Practice Guidelines in Oncology. J Natl Compr Canc Netw. 2016; 14: 882-913.

15. Atiq F, Hameli E, Broers AEC, Doorduijn JK, Van Gelder T, Andrews LM, et al. Converting cyclosporine A from intravenous to oral administration in hematopoietic stem cell transplant recipients and the role of azole antifungals. Eur J Clin Pharmacol. 2018; 74: 767-73.

16. Kolb M, Offer K, Jin Z, Kahn J, Bhatia M, Kung AL, et al. Risk Factors for Subtherapeutic Tacrolimus Levels after Conversion from Continuous Intravenous Infusion to Oral in Children after Allogeneic Hematopoietic Cell Transplantation. Biol Blood Marrow Transplant. 2016; 22: 957-61.

17. Yakoub-Agha I, Chabannon C, Bader P, Basak GW, Bonig H, Ciceri $\mathrm{F}$, et al. Management of adults and children undergoing chimeric antigen receptor T-cell therapy: best practice recommendations of the European Society for Blood and Marrow Transplantation (EBMT) and the Joint Accreditation Committee of ISCT and EBMT (JACIE). Haematologica. 2020; 105: 297-316.

18. Taplitz RA, Kennedy EB, Bow EJ, Crews J, Gleason C, Hawley DK, et al. Antimicrobial Prophylaxis for Adult Patients With Cancer-Related Immunosuppression: ASCO and IDSA Clinical Practice Guideline Update. J Clin Oncol. 2018; 36: 3043-54.

19. Network NCC. NCCN Clinical Practice Guidelines in Oncology (NCCN Guidelines ${ }^{\circledR}$ ) for Prevention and Treatment of Cancer-Related Infections. 2020.

20. Mahmoudjafari Z, Hawks KG, Hsieh AA, Plesca D, Gatwood KS, Culos KA. American Society for Blood and Marrow Transplantation Pharmacy Special Interest Group Survey on Chimeric Antigen Receptor T Cell Therapy Administrative, Logistic, and Toxicity Management Practices in the United States. Biol Blood Marrow Transplant. 2019; 25: 26-33.

21. Marr KA, Leisenring W, Crippa F, Slattery JT, Corey L, Boeckh M, et al. Cyclophosphamide metabolism is affected by azole antifungals. Blood. 2004; 103: 1557-9.

22. (Tisagenlecleucel) K. Product Information. Novartis Pharmaceuticals Corporation, United States; 2018.

23. Galli E, Allain V, Di Blasi R, Bernard S, Vercellino L, Morin F, et al. G-CSF does not worsen toxicities and efficacy of CAR-T cells in refractory/relapsed B-cell lymphoma. Bone Marrow Transplant. 2020; 55: 2347-9.

24. Hill JA, Giralt S, Torgerson TR, Lazarus HM. CAR-T - and a side order of IgG, to go? - Immunoglobulin replacement in patients receiving CAR-T cell therapy. Blood Rev. 2019; 38: 100596. 
25. Kristeller J. Transition of Care: Pharmacist Help Needed. Hosp Pharm. 2014; 49: 215-6.

26. Clemmons A. The Haematopoietic Cell Transplant Pharmacist: A Call to Action. Pharmacy. 2020; 8; doi:10.3390/pharmacy8010003.

27. Lehrer J, Brissot E, Ruggeri A, Dulery R, Vekhoff A, Battipaglia $\mathrm{G}$, et al. Medication adherence among allogeneic hematopoietic stem cell transplant recipients: a pilot singlecenter study. Bone Marrow Transplant. 2018; 53: 231-3.

28. Morrison CF, Martsolf DM, Wehrkamp N, Tehan R, Pai ALH. Medication adherence in hematopoietic stem cell transplantation: a review of the literature. Biol Blood Marrow Transplant. 2017; 23: 562-8.

29. Charra F, Philippe M, Herledan C, Caffin AG, Larbre V, Baudouin $\mathrm{A}$, et al. Immunosuppression medication adherence af- ter allogeneic hematopoietic stem cell transplant: Impact of a specialized clinical pharmacy program. J Oncol Pharm Pract. 2021; doi: 10.1177/10781552211000115.

30. Xu LP, Wu DP, Han MZ, Huang H, Liu QF, Liu DH, et al. A review of hematopoietic cell transplantation in China: data and trends during 2008-2016. Bone Marrow Transplant. 2017; 52: 1512-18. doi: 10.1038/bmt.2017.59.

https://doi.org/10.31547/bct-2021-016

Copyright (C)2021 Asia-Pacific Blood and Marrow Transplantation Group (APBMT). This is an open access article distributed under CC BY-NC license (https://creativecommon s.org/licenses/by-nc/4.0/). 